\title{
Disease burden of hepatitis $E$ in a rural population in China: a community-based survey
}

\author{
H. JIANG ${ }^{1} \dagger$, S. HUANG ${ }^{2} \dagger, Y . Z_{H A O}^{3} \dagger, Y . \mathrm{WANG}^{1}, \mathrm{C} . \mathrm{YANG}^{1}, \mathrm{~J} \mathrm{CAI}^{1}$, \\ Z. WANG ${ }^{1}$, J. ZHANG ${ }^{2}, X . Z_{H A N G}^{4} *$ AND H. JIN ${ }^{3}$ \\ ${ }^{1}$ Dongtai Center for Disease Control and Prevention, Dongtai, Jiangsu Province, China \\ ${ }^{2}$ State Key Laboratory of Molecular Vaccinology and Molecular Diagnostics, National Institute of Diagnostics \\ and Vaccine Development in Infectious Diseases, School of Public Health, Xiamen University, Xiamen, China \\ ${ }^{3}$ Key Laboratory of Environmental Medicine Engineering, Ministry of Education, School of Public Health, \\ Southeast University, Nanjing, China \\ ${ }^{4}$ Jiangsu Provincial Center for Disease Control and Prevention, Nanjing, China
}

Received 6 July 2016; Final revision 16 November 2016; Accepted 17 November 2016; first published online 19 January 2017

\section{SUMMARY}

This study aimed to estimate the disease burden of hepatitis $\mathrm{E}$ in a rural region in China. A total of 489 hepatitis E cases were reported according to a community-based survey in an active hepatitis surveillance system between 2008 and 2015, the questionnaire and record-review methods were constructed to evaluate the economic and health burden of hepatitis $\mathrm{E}$ virus infections from societal perspectives. All costs were converted to US\$ in 2015. The agestandardized cumulative incidence rate was $107 \cdot 9 / 100000$, and the median age-standardized annual incidence rate was $16 \cdot 5 / 100000$. The median direct, indirect, and intangible cost were $\$ 1046 \cdot 0, \$ 49 \cdot 1$, and $\$ 77 \cdot 3 /$ patient, respectively, and the median economic burden per patient was $\$ 1836 \cdot 5$, which accounted for $51 \cdot 2 \%$ of per capita disposable income. Moreover, the median quality-adjusted life year and visual analogue scale score were 0.7 and $70.0 /$ case, respectively. Both economic burden and health burden of inpatients was more serious than that of outpatients $(P<0.001)$. Disease burden of hepatitis $\mathrm{E}$ is heavy on patients, their families, and society. More studies on the disease burden of hepatitis $\mathrm{E}$ are necessary to increase social awareness of the disease and confirm reasonable disease-control measures.

Key words: Community-based survey, disease burden, hepatitis E.

\section{INTRODUCTION}

Hepatitis E, caused by hepatitis E virus (HEV), is an important public health concern as a common cause of enterically transmitted hepatitis worldwide. It is usually self-limiting but may develop to fulminant

\footnotetext{
* Author for correspondence: Mr X. Zhang, Jiangsu Provincial Center for Disease Control and Prevention, Nanjing, China. (Email: njzhangxf@126.com)

$\uparrow$ These authors contributed equally to this work.
}

hepatitis, especially in the pregnant population $[1,2]$. Two billion people, representing one third of the world's population, have been exposed to the virus [3, 4]; and according to Rein et al.'s estimate, there are 20 million HEV-infected cases, 3.4 million symptomatic cases, 70000 deaths, and 3000 stillbirths in nine endemic regions [5].

Estimating the disease burden of hepatitis $\mathrm{E}$ is important to increase scientific and social awareness of the disease and to inform decisions about health policy priorities and disease-control technologies [6], 
such as vaccines [7]. However, data on the disease burden are scarce [8, 9]. Therefore, we conducted a community-based study to evaluate the disease burden of hepatitis E cases reported between 2008 and 2015 in a rural region of China.

\section{METHODS}

\section{Hepatitis surveillance}

An active hepatitis surveillance system was enacted between 2008 and 2015 in 11 townships (Anfeng, Fuan, Hougang, Liangduo, Nanshenzao, Qindong, Shiyan, Tangyang, Wulie, Xinjie, Xuhe) of Dongtai City, Jiangsu Province, China, and the annual numbers of registered residents recruited in the surveillance system between 2008 and 2015 were 486417 , 483685, 482342, 478734, 473260, 471470, 466160, and 462813 , respectively. The working procedure of the surveillance system has already been discussed in previous studies, and a hepatitis $\mathrm{E}$ case was defined as a patient who fulfilled three conditions $[10,11]$ : acute illness lasting for at least 3 days; abnormal serum alanine transaminase concentration $\geqslant 2.5$ times the upper limit of normal range; and positive HEV IgM and RNA, $\geqslant 4$ times increase in HEV IgG, or both. Further, all hepatitis E cases reported in the active surveillance system were investigated face to face, and written informed consent was obtained from each participant before conducting research. Approval of the study was obtained from the Ethics Committee of the Jiangsu Provincial Center for Disease Control and Prevention.

\section{Prospective survey}

All participants were interviewed using a uniform questionnaire to collect information on basic demographic characteristics, economic burden, and health burden, and admission record review was used to assess inpatients' direct medical cost and expense afforded by medical insurance.

Demographic characteristics. Demographic characteristics were gender, age, degree of education, and profession, among others.

Economic burden. Economic burden included direct, indirect, and intangible economic burden. Direct economic burden was regarded as the sum of direct and indirect medical cost. Direct medical cost comprised expenses of outpatient services/visits, pharmaceutical drugs, nursing care, and other factors directly related to diagnosis and treatment; indirect medical cost covered transportation, nutritional services, and others [12]. Indirect economic burden monetarily assessed productivity loss of patients and individuals caring for patients [13]; however, we assumed that patients aged $>65$ years and their parents, wives/husbands, or friends caring for them did not lose work time due to being retired. The indirect economic burden was equal to disposable income per capita in Dongtai City in $2014(365 \cdot 25 \times$ productivity loss of the patient and individuals caring for the patient). We assumed that the value of disposable income per capita in Dongtai City in 2014 was approximately equal to that in 2015; intangible economic burden was calculated by converting 'disvalue' to an individual from pain, anxiety, and fear to monetary values based on the willingness-to-pay method [14, 15]. Moreover, Consumer Price Index (CPI) values obtained from the website of Yancheng City Bureau of Statistics were used to convert direct and intangible costs that occurred in other years into $2015 \mathrm{RMB}$, and RMB was converted to US dollars (\$) with an exchange rate of 6.2284 based on data from the National Bureau of Statistics of China.

Health burden. The EQ-5D-3L Chinese version was used to evaluate health burden of hepatitis E patients [16], EQ-5D-3L includes the descriptive system and the visual analogue scale (EQ-VAS) [17]. Qualityadjusted life year (QALY) and VAS score were indices of health burden. QALYs were gained from the conversion table for Japan (EQ-5D-JP) using time trade-off (TTO)-based preference scores [18].

\section{Statistical analysis}

EpiData v. 3.1 (EpiData Association, Denmark) and SPSS v. 19.0 (IBM Corp., USA) were used for data management and analysis. Incidence rate was standardized using the age data of the rural population in the Sixth National Population Census provided by The National Bureau of Statistics of China. Missing data of economic burden was completed with the median data, we assumed that outpatients expended $\$ 0$ where questions concerning economic burden were left blank; economic burden of inpatients was completed by the median cost of subjects with the same hospital and hospitalization time. Median and interquartile range (IQR) were used to describe concentrative and discrete trends of quantitative data. Wilcoxon and Kruskal-Wallis $H$ rank tests were used to compare disease burden between categorical subgroups when the 


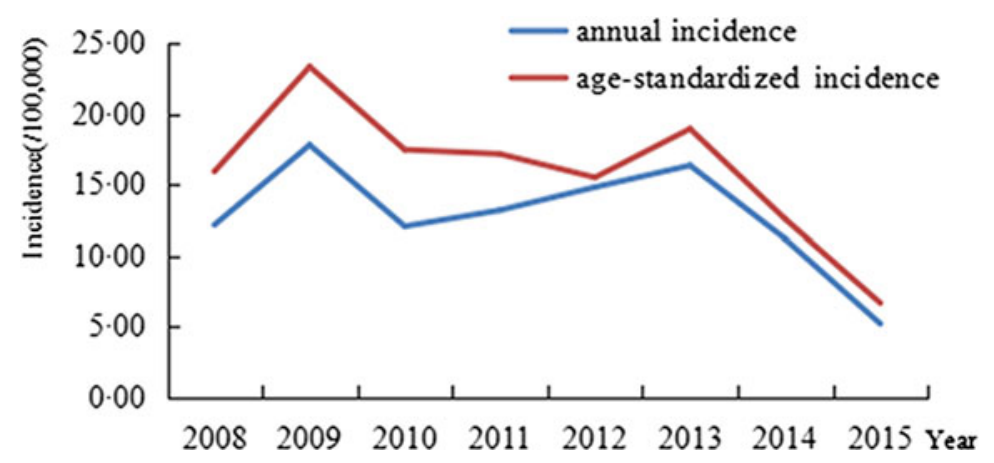

Fig. 1. Incidence rate of hepatitis $\mathrm{E}$ in a rural region of China. The $\mathrm{x}$-axis corresponds to the onset time (year), the $\mathrm{y}$-axis corresponds to the annual incidence rate.

outcome is not normally distributed. $P<0.001$ was considered statistically significant.

\section{RESULTS}

\section{Incidence of hepatitis $\mathrm{E}$}

A total of 489 hepatitis $\mathrm{E}$ cases were investigated in the active hepatitis surveillance system between 2008 and 2015. The eight years' cumulative incidence rate was $102 \cdot 7 / 100000$, and the age-standardized cumulative incidence rate was $107 \cdot 9 / 100000$. Moreover, the median annual incidence rate was $12 \cdot 6 / 100000$, and the median age-standardized annual incidence rate was $16 \cdot 5 / 100000$ (Fig. 1).

\section{Characteristics of hepatitis $\mathbf{E}$ patients}

The median age of hepatitis E patients was 58.0 (IQR $48 \cdot 5-67 \cdot 0)$ years. The male-to-female ratio was $2 \cdot 3: 1$, and $67.9 \%(332 / 489)$ of HEV-infected patients were admitted to hospital for treatment. Moreover, 75.3\% (368/489) of patients were farmers, $84 \cdot 5 \%$ (413/489) of infected patients had a low level of school education (no more than primary school education; grades $1-9)$, and $93 \cdot 5 \%$ (457/489) of cases were involved in the New Rural Cooperative Medical Scheme.

\section{Economic burden (Table 1)}

Direct economic burden. The median direct medical cost was $\$ 818 \cdot 8 /$ case, and the median direct medical cost for an outpatient and inpatient were $\$ 19 \cdot 3$ and $\$ 1813 \cdot 8$, respectively; cost of prescription drugs accounted for $70 \cdot 4 \%$ of hospitalization expenses, and $54.8 \%$ of hospitalization expenses was reimbursed by medical insurance. Moreover, the median indirect medical cost for an outpatient, inpatient, and hepatitis E case were $\$ 0 \cdot 7, \$ 241 \cdot 7$, and $\$ 110 \cdot 1$, respectively, and
$82.6 \%$ of indirect medical costs came from nutritional services. The median direct economic burden for one patient was $\$ 1046 \cdot 0$, which accounted for $29 \cdot 2 \%$ of disposable income per capita in Dongtai city. The ratio of direct medical cost to indirect medical cost was $7 \cdot 4: 1$, and there were statistically significant differences between gender and types of cases.

Indirect economic burden. The median duration of work loss was 5 days/case, and the median hospitalization time of inpatients was 16.5 days. The median indirect cost for one HEV-infected case was \$49.1, which accounted for $1.4 \%$ of disposable income per capita in Dongtai city. There was a significantly higher number of inpatients than outpatients; moreover, the cost of indirect economic burden differed statistically significantly in age groups.

Intangible economic burden. The median intangible cost was $\$ 77 \cdot 3 /$ case, which accounted for $2 \cdot 2 \%$ of disposable income per capita in Dongtai city. There were statistically significant differences between inpatients and outpatients.

In total, the median economic burden in a patient infected with HEV was $\$ 1836 \cdot 5$, which accounted for $51.2 \%$ of disposable income per capita in Dongtai city. The economic burden of inpatients was significantly higher than that of outpatients. Further, a multivariate linear regression model indicated inpatient/outpatient and age were significant factors for economic burden (gender, age, onset time, inpatient/outpatient and township were included in the analysis) $(P<0 \cdot 001)$.

\section{Health burden (Table 2)}

The median QALY and VAS score were $0 \cdot 7$ and $70 \cdot 0 /$ patient, respectively. Moreover, QALY and VAS scores 
Table 1. Economic burden for hepatitis $E$

\begin{tabular}{|c|c|c|c|c|c|}
\hline Variable & Number & Direct cost (US\$) & Indirect cost (US\$) & Intangible cost (US\$) & Total economic burden (US\$) \\
\hline \multicolumn{6}{|l|}{ Gender } \\
\hline Male & 339 & $1549 \cdot 2(596 \cdot 7-3165 \cdot 7)$ & $157 \cdot 0(0 \cdot 0-726 \cdot 3)$ & $87 \cdot 7(25 \cdot 4-371 \cdot 8)$ & $2707 \cdot 0(833 \cdot 0-4564 \cdot 2)$ \\
\hline Female & 150 & $373 \cdot 4(16 \cdot 5-1392 \cdot 2)$ & $19 \cdot 6(0 \cdot 0-316 \cdot 5)$ & $43 \cdot 9(17 \cdot 5-113 \cdot 3)$ & $636 \cdot 8(96 \cdot 1-2087 \cdot 0)$ \\
\hline$P^{*}$ & & $<0.001$ & 0.018 & 0.001 & 0.001 \\
\hline \multicolumn{6}{|l|}{ Age (years) } \\
\hline $15-29$ & 13 & $107 \cdot 4(4 \cdot 2-2152 \cdot 7)$ & $98 \cdot 1(4 \cdot 9-520 \cdot 2)$ & $49 \cdot 9(0 \cdot 0-92 \cdot 9)$ & $297 \cdot 3(57 \cdot 1-3481 \cdot 4)$ \\
\hline $30-44$ & 64 & $599 \cdot 8(20 \cdot 0-2340 \cdot 0)$ & $201 \cdot 2(12 \cdot 3-726 \cdot 3)$ & $79 \cdot 6(27 \cdot 8-316 \cdot 3)$ & $1530 \cdot 8(114 \cdot 7-4115 \cdot 4)$ \\
\hline $45-59$ & 189 & $1182 \cdot 1(90 \cdot 6-2883 \cdot 1)$ & $392 \cdot 6(44 \cdot 2-986 \cdot 4)$ & $68 \cdot 9(32 \cdot 1-305 \cdot 5)$ & $2523 \cdot 7(271 \cdot 3-4665 \cdot 2)$ \\
\hline$\geqslant 60$ & 223 & $1133 \cdot 5(492 \cdot 8-3076 \cdot 2)$ & $0 \cdot 0(0 \cdot 0-58 \cdot 9)$ & $83 \cdot 2(16 \cdot 6-193 \cdot 3)$ & $1669 \cdot 3(596 \cdot 7-3790 \cdot 9)$ \\
\hline$P_{\dagger}^{\dagger}$ & & 0.030 & $<0.001$ & $0 \cdot 282$ & 0.030 \\
\hline \multicolumn{6}{|l|}{ Onset period } \\
\hline 2008-2010 & 203 & $981 \cdot 4(302 \cdot 8-1943 \cdot 5)$ & $19 \cdot 6(0 \cdot 0-1060 \cdot 0)$ & $99 \cdot 6(55 \cdot 8-386 \cdot 6)$ & $1736 \cdot 3(591 \cdot 5-3847 \cdot 8)$ \\
\hline 2011-2015 & 286 & $1455 \cdot 7(69 \cdot 7-3170 \cdot 0)$ & $68 \cdot 7(0 \cdot 0-559 \cdot 4)$ & $43 \cdot 1(16 \cdot 6-88 \cdot 0)$ & $2045 \cdot 9(186 \cdot 9-4302 \cdot 1)$ \\
\hline$P^{*}$ & & $0 \cdot 030$ & $0 \cdot 874$ & $0 \cdot 001$ & $0 \cdot 920$ \\
\hline \multicolumn{6}{|c|}{ Types of cases } \\
\hline Outpatients & 167 & $23 \cdot 5(0 \cdot 7-167 \cdot 0)$ & $0 \cdot 0(0 \cdot 0-39 \cdot 3)$ & $35 \cdot 5(6 \cdot 9-92 \cdot 9)$ & $116 \cdot 0(39 \cdot 4-487 \cdot 8)$ \\
\hline Inpatients & 322 & $2235 \cdot 3(1001 \cdot 0-3420 \cdot 3)$ & $343 \cdot 5(0 \cdot 0-917 \cdot 7)$ & $92 \cdot 9(32 \cdot 1-386 \cdot 6)$ & $3272 \cdot 1(1689 \cdot 2-4932 \cdot 5)$ \\
\hline$P^{*}$ & & $<0.001$ & 0.001 & $<0.001$ & $<0.001$ \\
\hline Total & 489 & $1046 \cdot 0(107 \cdot 3-2821 \cdot 1)$ & $49 \cdot 1(0 \cdot 0-657 \cdot 6)$ & $77 \cdot 3(19 \cdot 3-199 \cdot 1)$ & $1836 \cdot 5(320 \cdot 1-4131 \cdot 1)$ \\
\hline
\end{tabular}

Values given are median (interquartile range).

* Wilcoxon rank test was used to analyse economic burden.

$\dagger$ Kruskal-Wallis $H$ rank test was used to analyse economic burden.

Table 2. Health burden for hepatitis $E$

\begin{tabular}{|c|c|c|c|c|c|}
\hline Variable & Number & QALY & Rank test* & EQ-VAS score & Rank test* \\
\hline \multicolumn{6}{|l|}{ Gender } \\
\hline Male & 339 & $0 \cdot 7(0 \cdot 6-0 \cdot 8)$ & $Z=-2 \cdot 360$ & $65 \cdot 0(50 \cdot 0-80 \cdot 0)$ & $Z=-3 \cdot 433$ \\
\hline Female & 150 & $0.7(0.6-0.9)$ & $P=0 \cdot 018$ & $70 \cdot 0(56 \cdot 5-88 \cdot 0)$ & $P=0.001$ \\
\hline \multicolumn{6}{|l|}{ Age (years) } \\
\hline $15-29$ & 13 & $1 \cdot 0(0 \cdot 7-1 \cdot 0)$ & $\chi^{2}=17 \cdot 198$ & $85 \cdot 0(76 \cdot 5-90 \cdot 0)$ & $\chi^{2}=21 \cdot 248$ \\
\hline $30-44$ & 64 & $0.7(0.6-0.8)$ & $P=0.001$ & $71 \cdot 5(60 \cdot 0-83 \cdot 8)$ & $P<0.001$ \\
\hline $45-59$ & 189 & $0.7(0 \cdot 6-0 \cdot 8)$ & & $70 \cdot 0(60 \cdot 0-80 \cdot 0)$ & \\
\hline$\geqslant 60$ & 223 & $0 \cdot 7(0 \cdot 6-0 \cdot 8)$ & & $60 \cdot 0(47 \cdot 3-75 \cdot 0)$ & \\
\hline \multicolumn{6}{|l|}{ Onset period } \\
\hline 2008-2010 & 203 & $0.7(0 \cdot 6-0.8)$ & $Z=-1 \cdot 520$ & $65 \cdot 0(45 \cdot 0-75 \cdot 5)$ & $Z=-3 \cdot 015$ \\
\hline 2011-2015 & 286 & $0.7(0.6-0.8)$ & $P=0 \cdot 128$ & $70 \cdot 0(55 \cdot 0-80 \cdot 0)$ & $P=0.002$ \\
\hline \multicolumn{6}{|l|}{ Types of cases } \\
\hline Outpatients & 167 & $0 \cdot 8(0 \cdot 7-1 \cdot 0)$ & $Z=-10 \cdot 610$ & $80 \cdot 0(64 \cdot 5-90 \cdot 0)$ & $Z=-8 \cdot 406$ \\
\hline Inpatients & 322 & $0.7(0 \cdot 6-0 \cdot 7)$ & $P<0.001$ & $61 \cdot 5(50 \cdot 0-70 \cdot 0)$ & $P<0.001$ \\
\hline Total & 489 & $0.7(0 \cdot 6-0 \cdot 8)$ & & $70 \cdot 0(50 \cdot 0-80 \cdot 0)$ & \\
\hline
\end{tabular}

QALY, Quality-adjusted life years; VAS, visual analogue scale.

Values given are median (interquartile range).

* Wilcoxon rank test was used for binary categorical variables; Kruskal-Wallis $H$ rank test was used for multi-categorical variables.

had a positive correlation $\left(r_{s}=0.521, \quad P<0.001\right)$. Health burden was greater for inpatients than outpatients. The health burden in older patients aged $>60$ years was higher than for other ages (QALY: 0.7 vs. $0 \cdot 7, z=-3 \cdot 219, P=0 \cdot 001$; EQ-VAS score: $60 \cdot 0 v s$.
70.00, $z=-3 \cdot 962, P<0 \cdot 001)$. A multivariate linear regression model showed inpatient/outpatient to be significant factors for QALY and VAS score (gender, age, onset time, inpatient/outpatient and township were included in the analysis) $(P<0 \cdot 001)$. 


\section{DISCUSSION}

Hepatitis A and E are two types of enterically transmitted viral hepatitis; however, hepatitis A is well prevented and controlled by universal hepatitis A vaccination, and hepatitis $\mathrm{E}$ accounts for a higher proportion of acute viral hepatitis [19]. Apart from this, the prevalence of anti-HEV IgG in the population is lower than the level observed for hepatitis A [20]. Surveys on the disease burden of hepatitis E are useful for comprehending the severity of the disease and being aware of the necessity of prevention and control measures in order to make more reasonable and effective public health decisions.

From 2008 to 2015, 489 HEV-infected cases were reported in the active hepatitis surveillance system in Dongtai city, China. The median annual incidence rate was $12 \cdot 6 / 100000$, which was lower than in 2006-2007 [11], because a recombinant hepatitis E vaccine with long-term efficacy was used after August 2007 [10, 21]; additionally, the incidence in 2015, shown in Figure 1, was underestimated due to the uncompleted 1-year survey.

The median direct, indirect, intangible, and total economic burden were $\$ 1046 \cdot 0, \$ 49 \cdot 1, \$ 77 \cdot 3$, and $\$ 1836 \cdot 5 /$ case, respectively, which accounted for $29 \cdot 2 \%, 1 \cdot 4 \%, 2 \cdot 2 \%$, and $51 \cdot 2 \%$ of per capita disposable income. This suggests that more reasonable measures should be implemented to prevent and control hepatitis $\mathrm{E}$, because the economic burden of hepatitis $\mathrm{E}$ accounted for more than $50 \%$ of disposable income per capita. Moreover, direct medical cost accounted for $78.3 \%$ of direct economic burden, and $54.8 \%$ of hospitalization cost would be reimbursed through medical insurance systems [22-24], which is higher than in previous studies [8,9]. Thus, the scientific and effective health insurance systems are also an important factor in reducing the economic burden of hepatitis $\mathrm{E}$ from the patients' perspective.

In this study, EQ-5D-3L was used to measure the health burden of hepatitis E patients, and the median QALY and VAS score per patient were 0.7 and $70 \cdot 0$, respectively. QALY, which reflects the opinion of the general population, is calculated by health state using TTO valuation techniques, but VAS records the patient's own assessment of their health status, which reflects a minor change in the quality of life of patients [17]. The results of EQ-VAS might be unreliable in this survey because patients with a low level of education might encounter difficulties in adequately understanding the definition of VAS, which could cause a low correlation coefficient between QALY and VAS scores. However, comprehension of the questionnaire was increased based on rapid development of media and close attention to the individual's own health. Older adults make up the high-risk population for HEV genotype 4 infection [3, 25], and the proportion of older patients (aged $>60$ years) reached $45.6 \%$ in the survey; furthermore, the median health burden in older patients is higher than in those of other ages. Therefore, older adults represent one targeted population group to prevent and control hepatitis $\mathrm{E}$ by health decision makers.

There are some limitations to this study. First, participants were recruited from one hepatitis E-endemic rural region in China where unreported cases were not considered [26], which might not represent the disease burden of hepatitis $\mathrm{E}$ in other areas, such as Beijing and Nanjing cities. Second, epidemiological features of hepatitis E cases are not sufficiently shown due to insufficient demographic data for registered residents recruited in the active hepatitis surveillance system. Third, more scales, such as the SF-6D [27] and EQ-5D-5L [28] should be used to confirm the most reasonable tool to measure the health burden of hepatitis E patients.

Currently, studies on disease burden of viral hepatitis mainly focus on hepatitis A, B, and C [29, 30]; however, the hepatitis $\mathrm{E}$ vaccine sold in China is safe and effective [10, 21], an effective immunization strategy is worth considering to prevent and control hepatitis E, and studies on disease burden concerning hepatitis $\mathrm{E}$ would provide rational evidence for a dominant immunization strategy.

\section{ACKNOWLEDGEMENTS}

This study was supported by the National Natural Fund of China (81573258), the Science \& Technology Demonstration Project for Emerging Infectious Diseases Control and Prevention (BE2015714) and the Social Development Fund of Jiangsu Province (BE2013723).

\section{DECLARATION OF INTEREST}

None.

\section{REFERENCES}

1. Hamid SS, et al. Fulminant hepatic failure in pregnant women: acute fatty liver or acute viral hepatitis? Journal of Hepatology 1996; 25: 20-27. 
2. Banait VS, et al. Outcome of acute liver failure due to acute hepatitis $\mathrm{E}$ in pregnant women. Indian Journal of Gastroenterology 2007; 26: 6-10.

3. Khuroo MS. Hepatitis E: an emerging global diseasefrom discovery towards control and cure. Journal of Viral Hepatitis 2016; 23: 68-79.

4. Mirazo S, et al. Transmission, diagnosis, and management of hepatitis E: an update. Hepatic Medicine: Evidence and Research 2014; 6: 45-59.

5. Rein DB, et al. The global burden of hepatitis E virus genotypes 1 and 2 in 2005. Hepatology 2012; 55: 988-997.

6. Conteh L, Engels T, Molyneux DH. Socioeconomic aspects of neglected tropical diseases. Lancet 2010; 375: 239-247.

7. Wu T, et al. Hepatitis E vaccine development A 14-year odyssey. Human Vaccines \& Immunotherapeutics 2012; 8: $823-827$.

8. Xiu SX. Disease burden of hepatitis E and health economic evaluation of vaccine interventions in Dongtai Area. Southeast University, 2011.

9. Jiang M, et al. Economic burden of patients with hepatitis E and influencing factors in Yantai city, China. Chinese Journal of Epidemiology 2011; 32: 845-846.

10. Zhu FC, et al. Efficacy and safety of a recombinant hepatitis $\mathrm{E}$ vaccine in healthy adults: a large-scale, randomised, double-blind placebo-controlled, phase 3 trial. Lancet 2010; 376: 895-902.

11. Zhu FC, et al. Epidemiology of zoonotic hepatitis E: a community-based surveillance study in a rural population in China. PLoS ONE 2014; 9: e87154.

12. Xie Z, et al. Posthospital discharge medical care costs and family burden associated with osteoporotic fracture patients in China from 2011 to 2013. Journal of Osteoporosis 2015; 25: 1-6.

13. Tarricone R. Cost-of-illness analysis. What room in health economics? Health Policy 2006; 77: 51-63.

14. Ogwulu CB, et al. Exploring the intangible economic costs of stillbirth. BMC Pregnancy and Childbirth 2015; 15: 188.

15. Wundes A, et al. Contribution of intangible costs to the economic burden of multiple sclerosis. Journal of Medical Economics 2010; 13: 626-632.
16. EQ-5D. (http://www.euroqol.org/eq-5d-products/eq-5d31.html).

17. The EuroQol Group. EQ-5D-3L user guide: basic information on how to use the EQ-5D-3L instrument, 2015.

18. Tsuchiya A, et al. Estimating an EQ-5D population value set: the case of Japan. Health economics 2002; 11: $341-353$.

19. Zhang M, et al. Analysis on morbidity and mortality of viral hepatitis in China, 2004-2013. Chinese Journal of Epidemiology 2015; 36: 144-147.

20. Anon. Hepatitis E vaccine: WHO position paper, May 2015. Weekly Epidemiological Record 2015; 90: 185-200.

21. Zhang J, et al. Long-term efficacy of a hepatitis E vaccine. New England Journal of Medicine 2015; 372: 914-922.

22. Pan $\mathbf{J}$, et al. Benefit distribution of social health insurance: evidence from China's urban resident basic medical insurance. Health Policy and Planning 2016.

23. Meng Q, et al. Consolidating the social health insurance schemes in China: towards an equitable and efficient health system. Lancet 2015; 386: 1484-1492.

24. Mahmood MA, et al. Cooperative medical insurance and the cost of care in Shandong, PR China: perspectives of patients and community members. Asia-Pacific Journal of Public Health 2015; 27: 897-902.

25. Kamar N, et al. Hepatitis E. Lancet 2012; 379: $2477-$ 2488.

26. Jia Z, et al. Epidemiology of hepatitis E virus in China: results from the Third National Viral Hepatitis Prevalence Survey, 2005-2006. PLoS ONE 2014; 9: e110837.

27. Jin H, et al. Comparison between EQ-5D and SF-6D utility in rural residents of Jiangsu Province, China. PLoS ONE 2012; 7: e41550.

28. Jia YX, et al. Comparison between the EQ-5D-5L and the EQ-5D-3L in patients with hepatitis B. Quality of Life Research 2014; 23: 2355-2363.

29. Steinke DT, et al. Epidemiology and economic burden of viral hepatitis: an observational population based study. Gut 2002; 50: 100-105.

30. Shon C, et al. The economic burden of hepatitis A, B, and $\mathrm{C}$ in South Korea. Japanese Journal of Infectious Diseases 2016; 69: 18-27. 